significance of learning of his/her HIV status. The study incorporated the stress-buffering model to propose that the beneficial effects of religious social support will modify the association between threat appraisal and well-being for PLHIV in a palliative care setting. Wellbeing was assessed both as the participant's subjective report of their well-being, and their subjective report of their experience with bodily pain. Participants' subjective report of well-being was hypothesized to be inversely associated with threat appraisal, and positively associated with religious social support. Subjective experience with bodily pain was hypothesized to be directly associated with threat appraisal, and inversely associated with religious social support. It was further also hypothesized that religious social support modifies the impact of threat-appraisal on well-being such that higher levels of religious social support reduce the observed effect of threat appraisal. METHODS/STUDY POPULATION: This was a cross-sectional study using baseline data from a randomized clinical trial-the FACE palliative care study in Washington, DC (FACE: FAmily CEntered Advance Care Planning). Participants were PLHIV who received their HIV care from 5 Washington, DC hospital-based HIV-specialty clinics. The FACE 3000 study paired participants into dyads of patient and surrogate decision-maker. The patient is a PLHIV for whom the advanced care planning care study is geared. The surrogate decision-maker is considered the patient's healthcare proxy who agrees to honor and advocate for the patient's treatment preferences, if the patient were unable to communicate with the health care team directly. Some surrogates are HIV positive, however due to their role as the patient's healthcare proxy, some of their surveys contain different content from those of the patient's. Potentially eligible participants in the FACE study received a secondary screening to determine eligibility to ensure competency to participate in end-of-life decision making. For this analysis, only the patient data was used. RESULTS/ANTICIPATED RESULTS: Subjective wellbeing showed significant associations with total threat appraisal, and four threat appraisal sub-constructs. Those with lower threat appraisals reported higher values of well-being compared to those with higher threat appraisals. Results from the regression analysis indicated that only one of the threat appraisal sub-constructs was significantly associated with a participant's subjective experience of pain. Overall, religious social support did not seem to buffer the effect of threat appraisal on well-being or subjective experience of pain. Findings from this study suggest that subjective well-being is associated with cognitive threat appraisal and this finding could assist PLHIV and their caregivers in understanding the coping processes of HIV-infected people. DISCUSSION/SIGNIFICANCE OF IMPACT: Due to stigmatization, an HIV diagnosis can influence a person's physical, behavioral, psychological, and even spiritual health (McIntosh \& Rosselli, 2012). As a stressor, it can compromise immune function to worsen the effects of the infection, while mentally depressing an individual and contributing to adverse coping mechanisms (e.g. alcohol consumption, drug use) (McIntosh \& Rosselli, 2012). How someone copes with stress (threat appraisal) may contribute to health-promoting or health-damaging behaviors (Fife, Scott, Fineberg, \& Zwickl, 2008). Hence, the quality of life of those managing HIV/AIDS remains a pressing concern. Findings from this study suggest that Lazarus and Folkman's theoretical framework on the cognitive appraisal of threat could assist PLHIV and their caregivers in understanding the coping processes in PLHIV. For service providers, recognizing early threat appraisals and damaging coping mechanisms can be useful, especially for patients receiving an initial HIV diagnosis. For example, an understanding of the patient's HIV appraisal can provide insight into the barriers to optimal care and adherence to ART and, potentially, help to reduce these barriers (Anderson, 1995). Furthermore, with the advancements of HIV medication, living with HIV has become a chronic condition, though as a stressor, it also poses long-term effects on the psychopathology of an individual living with HIV(McIntosh \& Rosselli, 2012). Studies such as this study can help illuminate interventions aimed at reducing the psychological impact of HIV on a person's life. For example, support groups have been developed and structured to provide social support and have been demonstrated to increase the perceived well-being among PLHIV (Hyde, Appleby, Weiss, Bailey, \& Morgan, 2005). This has further expanded into the consideration of online-based support groups for PLHIV (Blackstock, Shah, Haughton, \& Horvath, 2015). In another light, but still within psychosocial interventions for managing HIV infection, mindfulness meditation has been used pervasively in studies assessing its use as an intervention to reduce depression and perceived stress in people living with HIV in order to increase both physical and psychological health (Moskowitz et al., 2015). Interventions, such as mindful meditation, have risen as we understand more about appraisal pathways and coping strategies (such as seeking social support), and how they influence both physiological and psychological responses (Moskowitz et al., 2015) to affect the health of a person. Therefore, longitudinal research aimed toward management of the psychological and social consequences of HIV is central to promoting an accurate understanding of the quality of life for PLWH (Anderson, 1995).

3258

\section{The Relationship Between the Severity of Influenza-Related Illness and Timing of Seasonal Influenza Vaccination in Hospitalized Patients with Influenza \\ Julia Haston ${ }^{1}$, Shikha Garg, Angela Campbell, Jill Ferdinands, Monica Farley and Evan Anderson \\ ${ }^{1}$ Emory University}

OBJECTIVES/SPECIFIC AIMS: The overall goal of this project is to determine whether timing of seasonal influenza vaccination affects the severity of illness in vaccinated individuals who are hospitalized with influenza. This will be assessed with the following aims: 1. To determine whether differences in demographic and clinical characteristics exist among patients with short duration between seasonal influenza vaccination and influenza-related hospitalization and those with longer duration. 2. To determine whether time between seasonal influenza vaccination and hospitalization is associated with the duration of influenza-related hospitalization. 3. To determine whether time between seasonal influenza vaccination and hospitalization is associated with the rate of influenzarelated ICU admission among patients hospitalized with influenza. 4. To determine whether time between seasonal influenza vaccination and hospitalization is associated with the rate of influenzarelated death among adults hospitalized with influenza. METHODS/ STUDY POPULATION: The Influenza Hospitalization Surveillance Network (FluSurv-NET) is a surveillance platform of influenza-related hospitalizations through the CDC Emerging Infections Program (EIP). FluSurv-NET conducts active surveillance for influenza-related hospitalizations of both children and 
adults in selected counties in California, Colorado, Connecticut, Georgia, Maryland, Michigan, Minnesota, Ohio, Oregon, New Mexico, New York, Tennessee, and Utah with a total catchment population of over 27 million people ( $\sim 9 \%$ of the US population). Using this platform, we will retrospectively evaluate four influenza seasons using FluSurv-NET data to look at the timing of influenza vaccination and severity of illness among patients with influenzarelated hospitalization. We will conduct a multivariate analysis to assess for differences in severe outcomes including duration of hospitalization, ICU admission, and death among patients with varying lengths of time between influenza vaccination and influenza-related hospitalization. Separate analyses will be performed among different age groups and influenza type/subtypes, as well as specific seasons as a surrogate for most common circulating strain. RESULTS/ANTICIPATED RESULTS: We hypothesize that patients with chronic medical conditions and those at the extremes of age will have a longer duration between vaccination and hospitalization as they are more likely to get vaccinated earlier. We also hypothesize that patients with longer duration between seasonal influenza vaccination and hospitalization will have a longer duration of hospitalization and a higher rate of other severe outcomes (e.g., ICU admission, death). Such data would suggest that immune protection wanes during the influenza season. DISCUSSION/ SIGNIFICANCE OF IMPACT: Limited data suggest that vaccineinduced influenza immunity may wane during the influenza season. It is not known whether the impact of influenza vaccination upon severity of disease might wane with increasing time between vaccination and influenza infection. In contrast to many previous studies evaluating vaccine effectiveness which have assessed medically-attended influenza illness as a primary outcome, our dataset is a large cohort of hospitalized patients which allows us to assess rare yet critical outcomes such as ICU admission and death. This study will also have a substantially larger amount of pediatric data than previous studies, which will provide the opportunity to determine whether timing of vaccination affects children and adults differently. Improving our understanding of whether influenza vaccine-induced protection might wane over time could ultimately impact U.S. influenza vaccination policy resulting in decreased morbidity and mortality attributed to influenza each season.

\section{Torsade de pointes/QT prolongation risks with antibiotics: A contemporary analysis of the FDA Adverse Event Reporting System}

Chengwen Teng ${ }^{1}$, Daryl Kevin S. Gaspar and Christopher Frei

${ }^{1}$ University of Texas Health Science Center San Antonio

OBJECTIVES/SPECIFIC AIMS: Macrolides, linezolid, imipenemcilastatin, fluoroquinolones, penicillin combinations, and ceftriaxone are known to be associated with Torsade de pointes/QT prolongation (TdP/QTP). Other antibiotics may also lead to TdP/QTP, but no study has systemically compared TdP/QTP risks of different antibiotics using recent data. Therefore, the objective of this study was to evaluate the association between TdP/QTP and antibiotics in recent years using the FDA Adverse Event Report System (FAERS). METHODS/STUDY POPULATION: FAERS reports from January 1, 2015 to December 31, 2017 were analyzed. The Medical Dictionary for Regulatory Activities (MedDRA) was used to identify TdP/QTP cases. We calculated the Reporting Odds Ratios (RORs) and corresponding 95\% confidence intervals (95\%CI) for the association between antibiotics and TdP/QTP. An association was considered to be statistically significant when the lower limit of the $95 \% \mathrm{CI}$ was greater than 1. RESULTS/ANTICIPATED RESULTS: A total of 2,042,801 reports (including 5,221 TdP/QTP reports) were considered, after inclusion criteria were applied. Macrolides had the greatest proportion of TdP/QTP reports, representing $2.9 \%$ of all macrolide reports. TdP/QTP RORs (95\%CI) for the antibiotics were (in descending order): macrolides 11.73 (9.74-14.12), linezolid 9.39 (6.45-13.68), amikacin 8.94 (4.22-18.92), imipenem-cilastatin 5.01 (2.38-10.56), fluoroquinolones 4.67 (3.96-5.52), penicillin combinations 3.52 (2.56-4.86), cephalosporins 1.90 (1.14-3.16), metronidazole 1.49 (0.74-2.99), vancomycin 1.26 (0.70-2.28), clindamycin 0.83 (0.27-2.58), trimethoprim-sulfamethoxazole 0.82 (0.31-2.18), and amoxicillin $0.57(0.18-1.78)$. DISCUSSION/SIGNIFICANCE OF IMPACT: This study confirms prior evidence for TdP-QTP risks with macrolides, linezolid, imipenem-cilastatin, fluoroquinolones, penicillin combinations, and cephalosporins. This study provides new evidence for TdP-QTP risks with amikacin. Macrolides had the highest TdP/QTP ROR among the antibiotics evaluated in this study.

3317

Translating simulation-based team leadership training into patient-centered outcomes

Rosemarie Fernandez ${ }^{1}$, Elizabeth D. Rosenman, Anne K. Chipman, Sarah Brolliar, Marie C. Vrablik, Anthony T Misisco, Jeff Olenick, James Grand, Colleen Kalynych, Steve W. J. Kozlowski and

Georgia T. Chao

${ }^{1}$ University of Florida Clinical and Translational Science Institute

OBJECTIVES/SPECIFIC AIMS: The objective of this research was to assess the clinical impact of simulation-based team leadership training on team leadership effectiveness and patient care during actual trauma resuscitations. This translational work addresses an important gap in simulation research and medical education research. METHODS/STUDY POPULATION: Eligible trauma team leaders were randomized to the intervention (4-hour simulation-based leadership training) or control (standard training) condition. Subject-led actual trauma patient resuscitations were video recorded and coded for leadership behaviors (primary outcome) and patient care (secondary outcome) using novel leadership and trauma patient care metrics. Patient outcomes for trauma resuscitations were obtained through the Harborview Medical Center Trauma Registry and analyzed descriptively. A one-way ANCOVA analysis was conducted to test the effectiveness of our training intervention versus a control group for each outcome (leadership effectiveness and patient care) while accounting for pre-training performance, injury severity score, postgraduate training year, and days since training occurred. Association between leadership effectiveness and patient care was evaluated using random coefficient modeling. RESULTS/ ANTICIPATED RESULTS: Sixty team leaders, 30 in each condition, completed the study. There was a significant difference in posttraining leadership effectiveness $[\mathrm{F}(1,54)=30.19, \mathrm{p}<.001, \eta 2=.36]$ between the experimental and control conditions. There was no direct impact of training on patient care $[\mathrm{F}(1,54)=1.0, \mathrm{p}=0.33$, $\eta 2=.02$ ]; however, leadership effectiveness mediated an indirect effect of training on patient care. Across all trauma resuscitations 